\title{
Adhesion-related small bowel obstruction: deep learning for automatic transition-zone detection by $\mathrm{CT}$
}

Quentin Vanderbecq ${ }^{1,2^{*}}$ (D), Roberto Ardon ${ }^{3}$, Antoine De Reviers ${ }^{3}$, Camille Ruppli ${ }^{3}$, Axel Dallongeville ${ }^{1}$, Isabelle Boulay-Coletta', Gaspard D'Assignies ${ }^{3}$ and Marc Zins ${ }^{1}$

\begin{abstract}
Background: To train a machine-learning model to locate the transition zone (TZ) of adhesion-related small bowel obstruction (SBO) on CT scans.

Materials and methods: We used 562 CTs performed in 2005-2018 in 404 patients with adhesion-related SBO. Annotation of the TZs was performed by experienced radiologists and trained residents using bounding boxes. Preprocessing involved using a pretrained model to extract the abdominopelvic region. We modeled TZ localization as a binary classification problem by splitting the abdominopelvic region into 125 patches. We then trained a neural network model to classify each patch as containing or not containing a TZ. We coupled this with a trained probabilistic estimation of presence of a TZ in each patch. The models were first evaluated by computing the area under the receiver operating characteristics curve (AUROC). Then, to assess the clinical benefit, we measured the proportion of total abdominopelvic volume classified as containing a TZ for several different false-negative rates.
\end{abstract}

Results: The probability of containing a TZ was highest for the hypogastric region (56.9\%). The coupled classification network and probability mapping produced an AUROC of 0.93 . For a $15 \%$ proportion of volume classified as containing TZs, the probability of highlighted patches containing a TZ was $92 \%$.

Conclusion: Modeling TZ localization by coupling convolutional neural network classification and probabilistic localization estimation shows the way to a possible automatic TZ detection, a complex radiological task with a major clinical impact.

Keywords: Abdomen, Small bowel, Obstruction, Neural networks, Tomography (X-ray computed)

\section{Key points}

- We combined CNN classification and probabilistic mapping to detect the transition zone of adhesion related SBO.

- Transition zones were most commonly located in the hypogastric region (56.9\%).

\footnotetext{
*Correspondence: q.vanderbecq@gmail.com

${ }^{1}$ Department of Medical Imaging, Saint Joseph Hospital, 185 rue Raymond Losserand, 75014 Paris, France

Full list of author information is available at the end of the article
}

- The coupled classification-network and probabilitymapping model produced an area under the ROC curve of 0.93 .

\section{Introduction}

Small bowel obstruction (SBO) is a common nontraumatic surgical emergency, with approximately 400,000 admissions annually in the United States [1]. Among causes of SBO, the most common are adhesions [2].

All guidelines recommend computed tomography (CT) as the first-line imaging study for patients with suspected 
mechanical SBO [3, 4]. The goal is four-fold: (i) to confirm or refute the diagnosis of SBO and, when SBO is present, (ii) to locate the site of the obstruction, that is, the transition zone (TZ) (iii) to identify the cause, and (iv) to look for complications such as strangulation or perforation. Identifying the $\mathrm{TZ}$ or TZs (and determining their number) and establishing their locations is the first step in diagnosing the cause of SBO and differentiating the open-loop and closed-loop mechanisms [5]. A diagnosis of closed-loop SBO independently predicts ischemia [6] and help to decide whether surgery is needed in patients with adhesion-related SBO.

However, identifying the TZ or TZs is time-consuming and subject to inter-observer and intra-observer variability [7]. Automated TZ localization would therefore prove valuable for expediting the diagnosis in emergency cases and improving radiologists' performance in diagnosing the closed-loop mechanism.

The potential of machine learning to contribute to radiological diagnoses has expanded at a brisk pace in recent decades, initially thanks to increases in data-storage capabilities and subsequently due to the advent of parallel-processing hardware based on graphical processing units [8]. As a result, the number of studies of deep neural networks in medical imaging is escalating sharply. However, few teams are focusing on SBO. The only published classifications models were produced for standard abdominal radiographs [9-11]. No studies have used CT or 3D models, despite the recognized benefits of CT for diagnosing SBO and the probable contribution of 3D models, which may be comparable to that of multiplanar reformation [12].

The objective of this study was to build a 3D deeplearning model to help radiologists locate the $\mathrm{TZ}$ or $\mathrm{TZs}$ on CT scans from patients with adhesion-related SBO.

\section{Material and methods Dataset}

All abdominopelvic CT scans performed at our institution between January 2005 and July 2018 were identified retrospectively. Of the 42,000 consecutive CT scan reports for the study period, 4098 contained the word or words "obstruction" and/or "intestine". Of these 4098 CTs, 2472 had a health information system code for SBO. An abdominal expert radiologist determined that 1287 of the 2472 reports were for patients with mechanical SBO. Finally, 562 CTs from 404 patients with adhesion-related SBO were identified and annotated. Median patient age was 70 years (range 16-90 years) and there were 312 women and 250 men. Additional file 1: Table S1 describes the distribution of the study CTs and Additional file 1: Text $\mathrm{T} 1$ the $\mathrm{CT}$ acquisition technique.
Of the 562 CTs, 247 were annotated by 7 radiologists with 2 to over 30 years of experience in abdominal CT. The remaining 315 CT scans were annotated by 9 residents who were previously trained in the process. All annotations were reviewed by study coordinator (Q.V.) and consensus has been reached with an expert with over 30 years of experience in abdominal radiology (M.Z.) for contentious cases between the study coordinator and radiologist annotator. These annotations were done using a platform built by Incepto-Medical (Paris, France). The annotators were asked to place one bounding box around a single TZ in case of open-loop obstruction and two bounding boxes for closed-loop obstruction [5]. Figure 1 summarizes the patient selection and reporting process.

Patients were randomly selected for training, validation, and testing, in proportions of $70 \%, 20 \%$, and $10 \%$, respectively. The split was performed at the patient level, with no overlap, i.e., volumes for a given patient appeared in only one of the three sets. Population parameters (age, sex) and annotator experience followed the same statistical distribution in all three sets (Additional file 1: Table S2).

\section{Preprocessing}

CT scans provide high-resolution images. Volumes in the database were 3D arrays of size (nslices; 512; 512), where nslices was between 300 and 500 . We applied the following preprocessing steps. First, a pretrained algorithm extracted the abdominopelvic region. Second, the random walk algorithm was used to segment the abdominopelvic region into a volume with a mean size of $300 \times 400 \times 300$, with minimal void around the body.

This preprocessing method provided each of the abdominopelvic volumes with an anatomical bounding box. For a given volume selected as the reference, we used affine transformation to register all other abdominopelvic bounding box volumes. We summed the contributions of each box to generate a heatmap associated with the reference volume. This heatmap indicated the spatial distribution of TZs.

To locate each TZ, we used a patch-based approach to train a binary classification model. We performed three different experiments, splitting the abdominopelvic region into $27(3 \times 3 \times 3), 64(4 \times 4 \times 4)$, or $125(5 \times 5 \times 5)$ patches. Each patch was classified in a binary manner, i.e., with or without a TZ, using the center of the annotated bounding box as the standard reference for TZ location. To train deep-learning classification models, each patch was resized to $64 \times 64 \times 64$.

\section{Model and training parameters}

We modeled a convolutional neural network (CNN) with four convolution blocks followed by two dense 


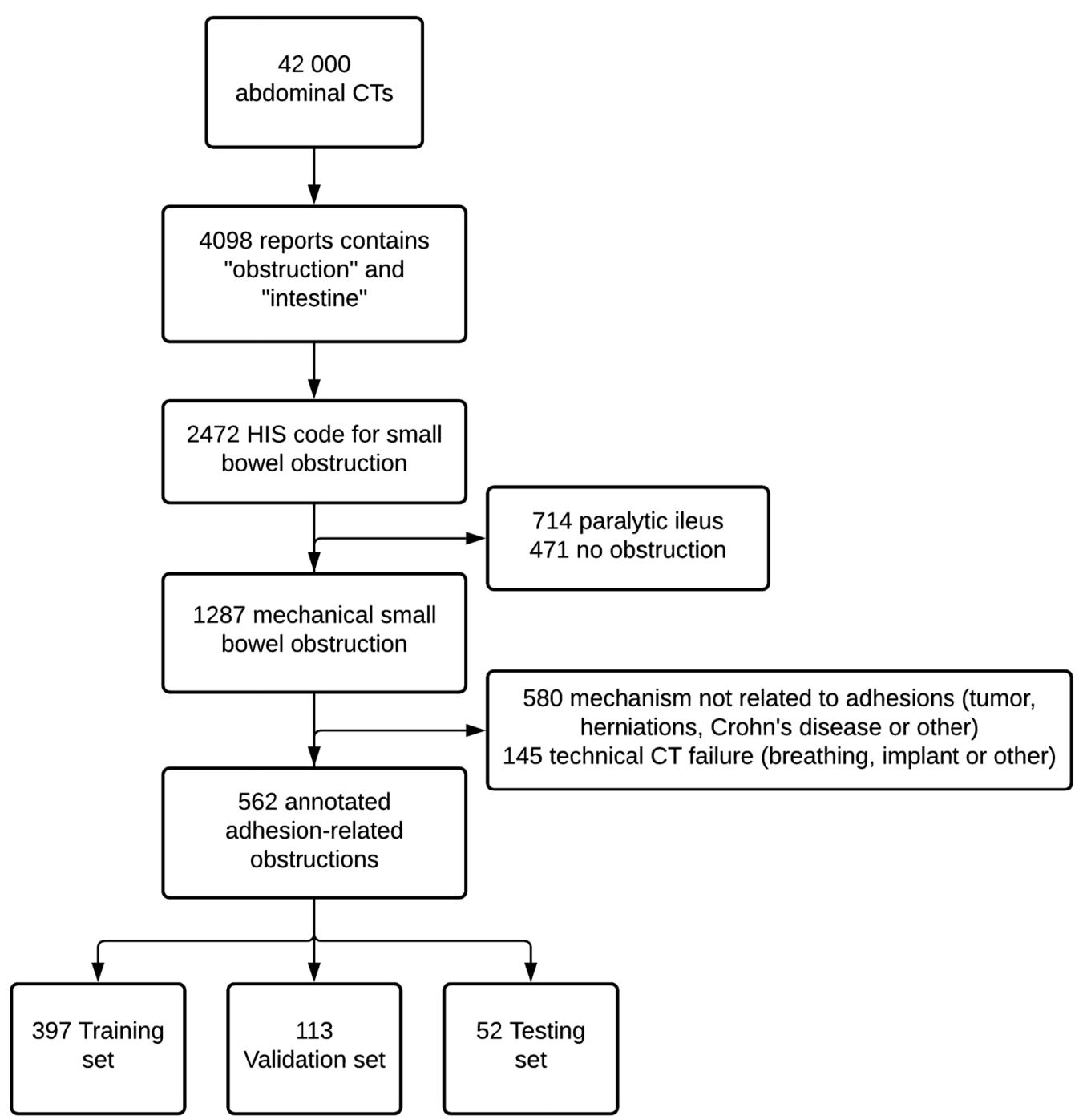

Fig. 1 Flow chart of the data selection process. HIS: health information system

layers (Fig. 2). Each convolution block was composed of two 3D convolution layers (kernel size 3) followed by Rectified Linear Unit (ReLU) activation and batch normalization then by a 3D MaxPooling layer (pool size 2). Each dense layer was followed by ReLU activation with a 0.5 dropout rate to prevent overfitting.

We used balanced cross entropy loss to increase the weights of positive sample classifications, thereby mitigating database imbalance. We also performed data augmentation on positive patches with random translation between 0 and $3 \mathrm{~cm}$ in each direction and random zoom between 0.8 and 1.2 .
Reduction of the search space for the transition zone (TZ) The output of our model was a map overlaid on the abdominopelvic region. On the map, areas most likely to contain a TZ were highlighted. We used two methods to generate this map. The first highlighted patches if the CNN classification score was above the threshold maximizing the Youden index (sensitivity + specificity - 1, computed on the validation database) [13]. The second method highlighted patches based on the CNN classification score multiplied by the patch spatial probability of containing a $\mathrm{TZ}$ (or TZs), calculated on our training set. 


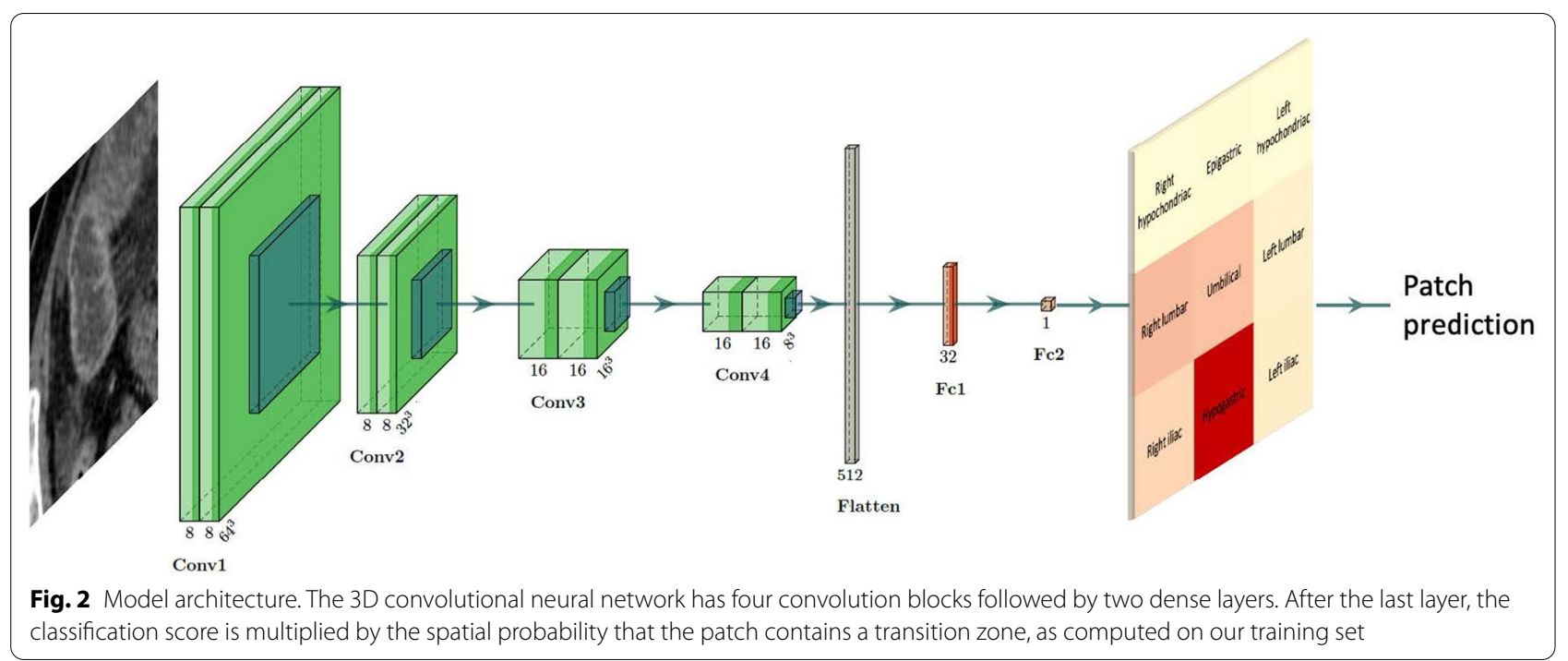

\section{Classification performance}

To compare classification models, we computed the area under the receiver operating curve (AUROC) and preci$\operatorname{sion}\left(\frac{\text { True positive }}{\text { True postive }+ \text { False positive }}\right)$.

\section{Search-space reduction versus risk of missing the transition zone (TZ)}

To evaluate the practical benefits of our method, we compared the proportion of abdominopelvic volume that was highlighted (using both above-described methods) to the probability of missing a TZ. This last probability was computed on the test database.

\section{Results}

\section{Transition zone (TZ) location}

We first analyzed our dataset of 562 annotated CT scans. As outlined above, each bounding box was registered in a selected body, and a heatmap was built by summing the contributions of each box (Fig. 3). Analysis of the abdominal quadrant demonstrated that TZs predominated in the hypogastric quadrant $(56.9 \%)$ and more than $85 \%$ of TZs are located in three regions (hypogastric, right lumbar and umbilical regions) (Table 1).

\section{Classification performance}

We used the training set to train the $\mathrm{CNN}$, the validation set to tune the optimization parameters (learning rate and stopping criteria of the training stage), and the test set for the final evaluation. We evaluated different abdomen partitions in $27(3 \times 3 \times 3$ partitions $)$, $64(4 \times 4 \times 4)$ and $125(5 \times 5 \times 5)$ patches. And we achieve an AUROC of 0.95 (CI: 0.92-0.97), 0.95 (CI: 0.93, 0.96), 0.93 (CI: 0.89-0.97) (Fig. 4) and a mean precision of 0.43 (CI: 0.30-0.57), 0.31 (CI: 0.19-0.45) and 0.13 (CI: $0.08-0.22$ ), respectively. The differences in these results are attributable to the dataset imbalance: because the prevalence of TZs across all patches of each patient was very low, both good sensitivity and good specificity were achieved but mean precision was very low (Additional file 1: Table S3).

Moreover, the AUROC values for patches positioned on specific anatomical regions varied widely (Fig. 5). For instance, the value was very high for the right lumbar region but was only 0.80 in the umbilical and hypogastric regions.

\section{Search-space reduction versus risk of missing the transition zone (TZ)}

To integrate this spatial heterogeneity, each patch classification score was weighted by its spatial probability of containing a TZ (Table 1 ).

The AUROC for our best weighted patch classification model (125 patches) was 0.93 . While this value may be considered acceptable, it was insufficient for TZ localization. To locate the TZ, 125 predictions were required, and an acceptable patch classifier would find a single positive patch. In our case, the false-positive rate (maximizing the Youden index) was 0.14.

Under the hypothesis of a single patch containing the $\mathrm{TZ}$, the number of highlighted patches that were erroneously classified followed the binomial law of parameters $(124,0.14)$. The mean number of erroneously classified patches was 17/124. We thus chose to exploit our classifier as a highlighting map identifying the abdominopelvic regions most likely to contain the $\mathrm{TZ}$. 


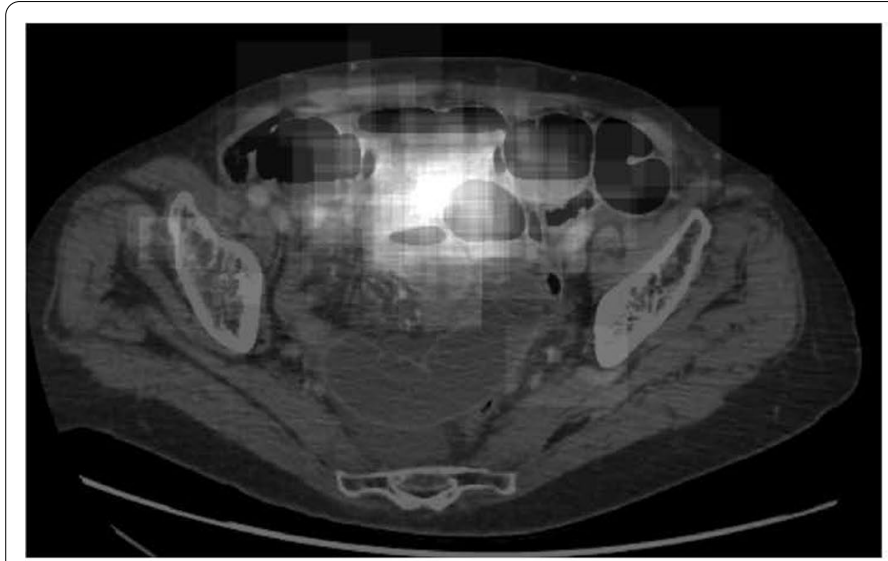

(a) Axial view
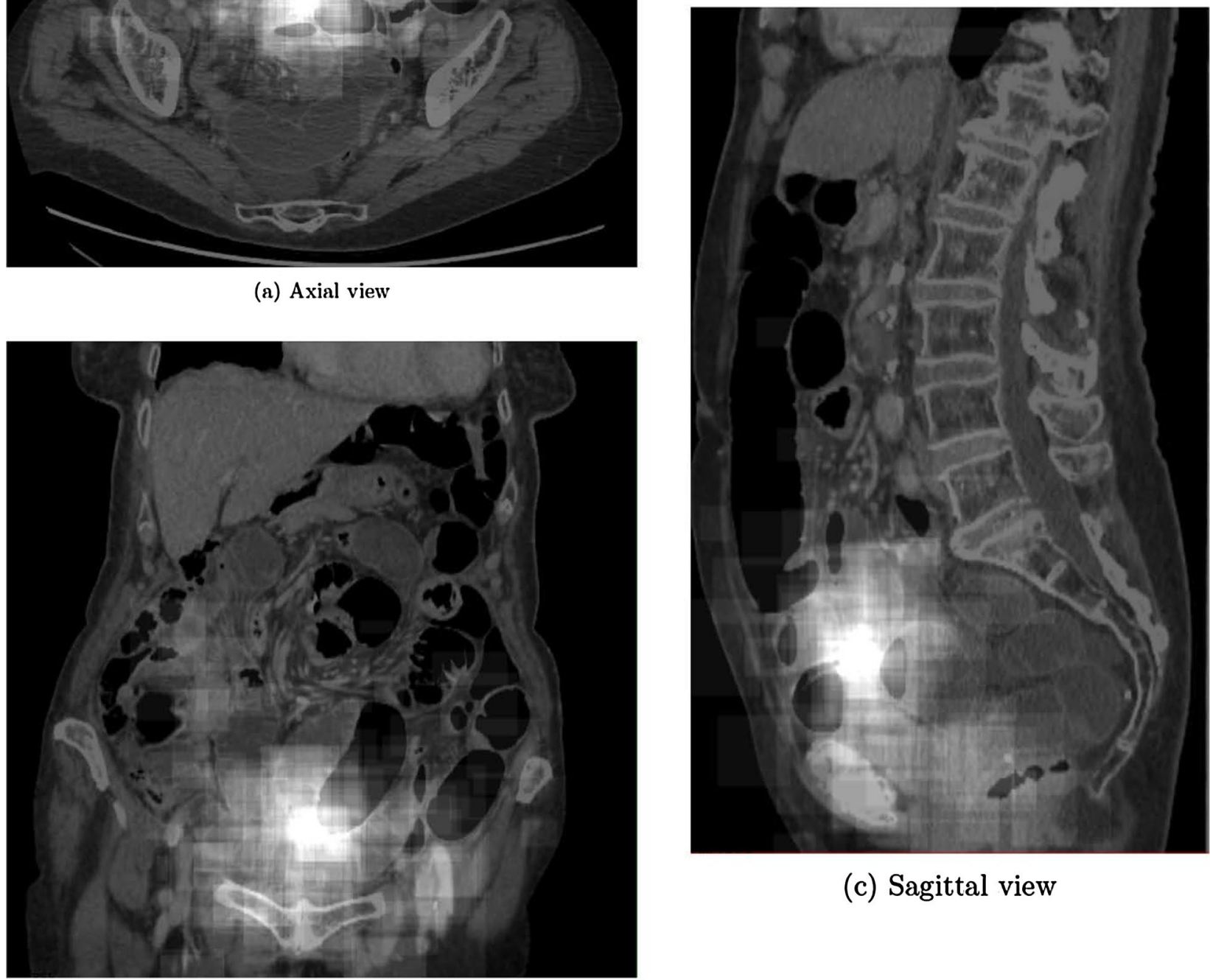

(c) Sagittal view

(b) Coronal view

Fig. 3 Heatmap of adhesion-related small bowel obstruction transition zone (TZ) localization. The images are shown with grey scale, with a maximum of $56.9 \%$ in white, to show the probability of presence of a TZ in any given reference volume

Table 1 Percentage of transition zones found in each of the six abdominal regions

\begin{tabular}{lllllllll}
\hline Right hypochondriac & Epigastric & Left hypochondriac & Right lumbar & Umbilical & Left lumbar & Right iliac & Hypogastric & Left iliac \\
\hline 0 & 0.3 & 0.2 & 14.7 & 13.1 & 2.8 & 8.5 & 56.9 & 3.4 \\
\hline
\end{tabular}

With spatial weighting, the proportion of highlighted abdominopelvic volume decreased while keeping an acceptable probability of missing the TZ (Fig. 6).
Figures 4 and 6 shows the impact of this spatial adjustment on the percentage of abdominopelvic volume highlighted by the model. The AUROC value improved only by 0.01 or 0.02 , depending on patch division (Fig. 5), with 

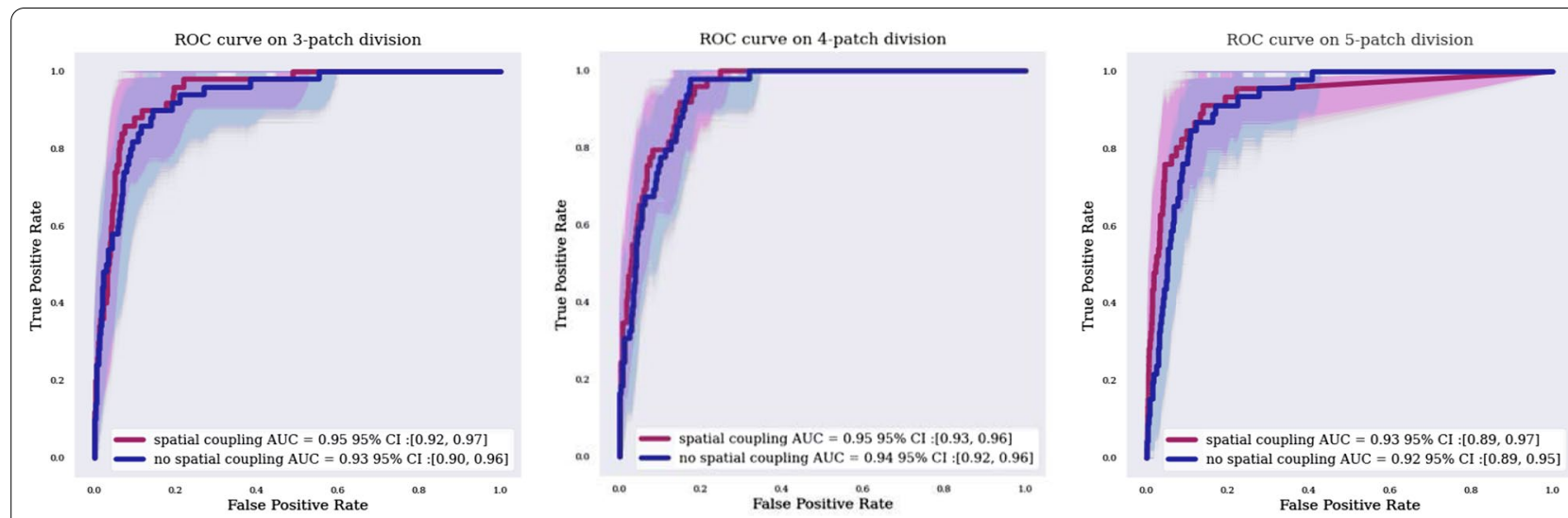

Fig. 4 AUROC curves obtained with the different divisions of the abdomino-pelvic region. Divisions into $3 \times 3 \times 3$ patches ( 3 -patch division, left), $4 \times 4 \times 4$ patches ( 4 -patch division, center), and $5 \times 5 \times 5$ patches (5-patch division, right)
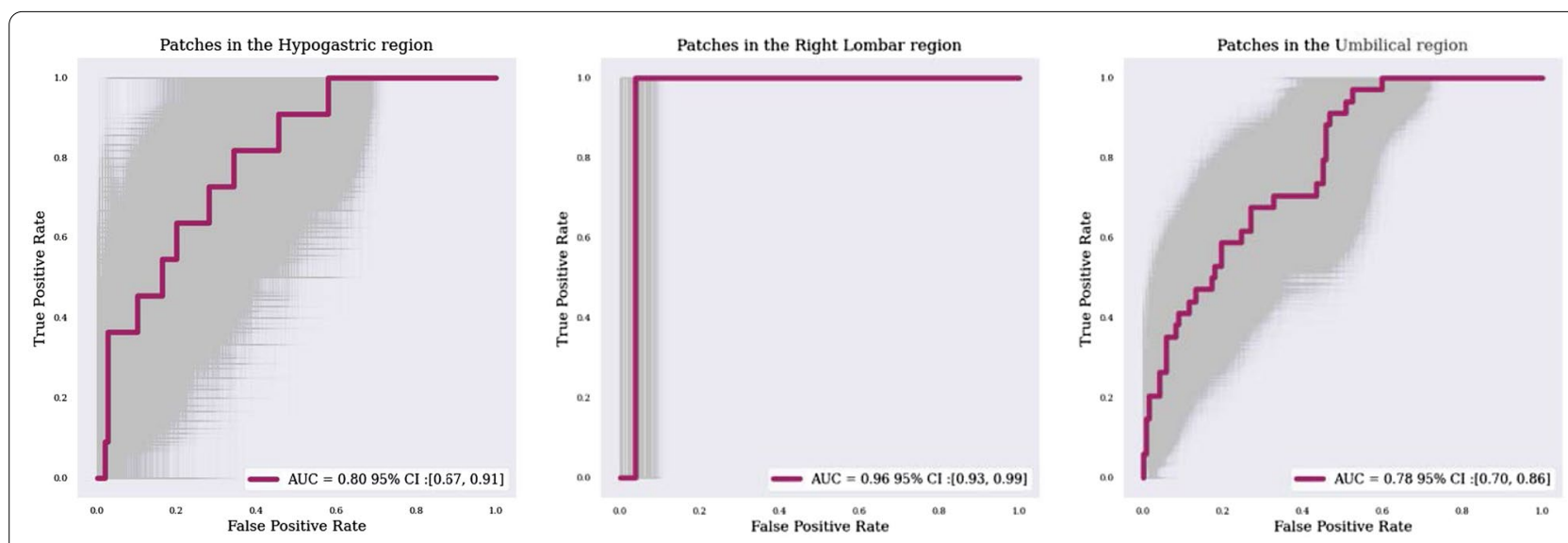

Fig. 5 AUROC curves in three specific abdominal regions: hypogastric (left), right lumbar (center), and umbilical (right). These curves were computed by summed the patch classification scores in hypogastric, lumbar and umbilical regions

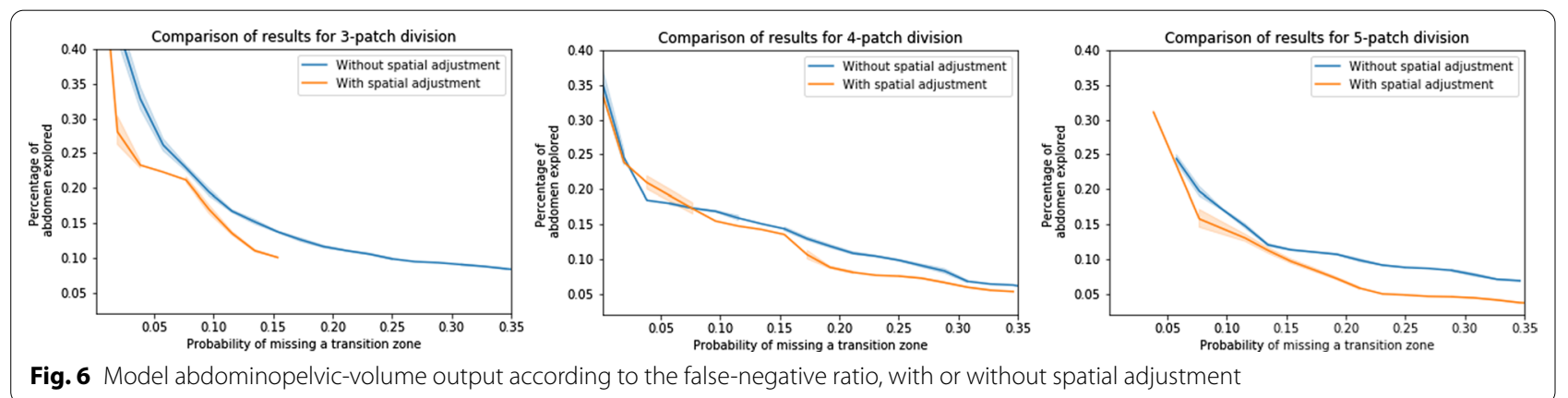

no change in the false-negative rate, but the searched abdominopelvic volume decreased by $4 \%$ (Fig. 6).
Our best map (125 patches with spatial coupling) highlighted the TZ with a probability of $93 \%$ while covering 
only $15 \%$ of the abdominopelvic volume. Additional file 1: Figure S1 shows an example of an output result.

\section{Discussion}

We developed a machine-learning model to reduce the TZ search space on abdominopelvic CT scans of patients with suspected SBO. To that end, we built a large annotated SBO database.

Several factors may explain why only two earlier studies have evaluated an artificial-intelligence approach to SBO diagnosis, despite the high frequency of mechanical SBO among nontraumatic surgical emergencies $[9,10]$. It should be noted that these studies focused on standard radiographs, which are no longer recommended for diagnosing SBO [4]. First, developing an artificial-intelligence model for $\mathrm{TZ}$ detection requires the establishment of a large database by highly experienced radiologists. Second, the abdominopelvic volume in which the TZ may reside is large compared to other medical volumes such as the intracranial space. Third, the abdominopelvic volume varies considerably across individuals due to differences in the amount of intra-abdominal fat and in intestinal motion. Finally, TZ detection is facilitated by a $3 \mathrm{D}$ assessment [12], which requires a more complex model than those used in radiography.

To precisely locate a transition zone, high resolution imaging is needed. Training a model using the whole CT volume at such resolution requires high performance GPUs. These GPUs are incompatible with routine clinical use because of their computational costs. To be able to train at high resolution we chose a balance between performance objective and technical limits and performed a patch extraction before classification as other detection networks do [14].

Locating the $\mathrm{TZ}$ is not only of diagnostic value but may also help to plan surgical procedures, for instance trocar placement for laparoscopy. In a recent metanalysis, CT was $92 \%$ sensitive and $87 \%$ specific for detecting the $\mathrm{TZ}$ [15]. However, this good performance comes at the cost of spending considerable radiologist time. $\mathrm{TZ}$ location is the first step toward establishing the cause of SBO and evaluating the risk of ischemia. Unfortunately, radiologist precision for detecting a closed-loop mechanism is poor. In a study of 88 patients who had surgery for SBO, including 24 with a closed-loop mechanism, sensitivity of CT interpreted by radiologists for detecting this mechanism was only $53 \%$ (95\% confidence interval, $44 \%-63 \%$ ) and specificity was $83 \%(79 \%-87 \%)$ [7]. Furthermore, inter-observer agreement for closed loop detection was poor to moderate $(k=0.39-0.63)$.

To facilitate TZ detection on CTs, we built a model designed to decrease the search volume in the abdominopelvic region, accelerate the emergency-CT workflow
$[16,17]$, and increase the appropriateness of treatment decisions. We expected TZs to predominate in the right iliac region, since a leading cause of adhesion-related $\mathrm{SBO}$ is prior abdominal surgery and appendectomy is a common procedure [18]. Unexpectedly, TZs predominated significantly in the hypogastric region. We are aware of a single study of TZ location, which was determined only in the sagittal plane [19]. The results showed that the closed-loop mechanism was most common when there was more than one $\mathrm{TZ}$ and the TZs were located posteriorly, within $7 \mathrm{~cm}$ anterior to the anterior edge of the spine. The predominance of $\mathrm{TZ}$ in hypogastric region may be related to the high frequency of gynecological surgical procedures. Furthermore, the appendix is sometimes located in the pelvis.

Coupling a deep-learning classification model with a priori spatial knowledge improved model performance in our study. Although this is a simple logical concept in medicine, few deep-learning models applied to the medical domain leverage it. Only a few recent models in the field of natural images have started to use spatial probability estimation [20].

A limitation of our study relates to external validity, since the data came from a single center and all the models of CT machines used were from the same manufacturer. This limitation applies chiefly to our testing set. Since the accuracy for identifying a patch containing a single TZ is limited, our model is not optimal for clinical practice use. Much work remains to be done to build machine-learning models that approach the performance of an abdominal imaging radiologist. We only studied adhesion-related SBO as they represent more than $60 \%$ of SBO etiologies [21]. A further study should include all etiologies. The data ground truth may be open to criticism: we relied on annotations made by specialists on CT scans as opposed to a biological reference standard such as those often available in other fields (e.g., oncology). Radiologists have demonstrated good sensitivity and specificity for TZ localization [15], despite the significant heterogeneity in this parameter. The best reference standard for TZ location by CT remains unclear. In particular we can assume that a surgical reference, could be optimal. However, an important proportion of adhesiverelated SBO are managed non operatively, and to obtain a representative population of bowel obstructions we cannot restrict our inclusions to operated SBO. Moreover, the possibility of precisely locating the junctional zone in space during surgery is subject to discussion.

Finally, to build a prediction model for the management of adhesion related SBO, the first step is to locate the TZ. This information helps to determine whether the mechanism is open or closed loop the latter being part of the risk factors for intestinal ischemia [19]. We now plan 
to develop models that predict ischemia, with annotation on CT scans of decreased bowel-wall enhancement and diffuse mesenteric haziness.

To conclude, our study makes two important contributions. First, the results show that the hypogastric region is the most common location of TZs in patients with adhesion related SBO. Second, our model combining $\mathrm{CNN}$ with a probabilistic mapping reduced the abdominopelvic TZ-search volume. This is a first step towards the development of an effective machine learning system that will save valuable time when performing complex and often urgent $\mathrm{TZ}$ identification and localization.

\section{Abbreviations}

AUROC: Area under the receiver operating characteristics curve; CNN: Convolutional neural network; CT: Computed tomography; SBO: Small bowel obstruction; TZ: Transition zone.

\section{Supplementary Information}

The online version contains supplementary material available at https://doi. org/10.1186/s13244-021-01150-y.

Additional file 1. Table S1: Distribution of the CT machines used. Table S2: Distribution of patient demographics and experience of annotator. Table S3: AUROC and precision results on the test set. Supplementary Text S1: Additional technical information. Figure S1: Example of result.

\section{Acknowledgements}

We thank Neesmah Badat, Anne-Marie Tardivel, Vincent Barrau, Alexia Tran, Thibaud Morcet, Axel Cohen, Paul-Gydeon Ritvo, Yoann Sclover, Dominique Ngouana, David Tob, and Charlotte Pouchy for their participation in annotating the dataset.

\section{Authors' contributions}

QV have made substantial contributions to the conception AND design of the work AND interpretation of data; AND have drafted the work or substantively revised it AND to have approved the submitted version (and any substantially modified version that involves the author's contribution to the study) AND to have agreed both to be personally accountable for the author's own contributions and to ensure that questions related to the accuracy or integrity of any part of the work, even ones in which the author was not personally involved, are appropriately investigated, resolved, and the resolution documented in the literature. RA have made substantial contributions to the conception and design of the work and design of the work; and the creation of new software used in the work; and have drafted the work or substantively revised it AND to have approved the submitted version (and any substantially modified version that involves the author's contribution to the study); AND to have agreed both to be personally accountable for the author's own contributions and to ensure that questions related to the accuracy or integrity of any part of the work, even ones in which the author was not personally involved, are appropriately investigated, resolved, and the resolution documented in the literature. ADR have made substantial contributions to the conception and the creation of new software used in the work and have drafted the work or substantively revised it AND to have approved the submitted version (and any substantially modified version that involves the author's contribution to the study); AND to have agreed both to be personally accountable for the author's own contributions and to ensure that questions related to the accuracy or integrity of any part of the work, even ones in which the author was not personally involved, are appropriately investigated, resolved, and the resolution documented in the literature. CR have made substantial contributions to the conception and the creation of new software used in the work and have drafted the work or substantively revised it AND to have approved the submitted version (and any substantially modified version that involves the author's contribution to the study); AND to have agreed both to be personally accountable for the author's own contributions and to ensure that questions related to the accuracy or integrity of any part of the work, even ones in which the author was not personally involved, are appropriately investigated, resolved, and the resolution documented in the literature. AD have made substantial contributions to the conception and design of the work; and the acquisition OR interpretation of data; AND to have approved the submitted version (and any substantially modified version that involves the author's contribution to the study); AND to have agreed both to be personally accountable for the author's own contributions and to ensure that questions related to the accuracy or integrity of any part of the work, even ones in which the author was not personally involved, are appropriately investigated, resolved, and the resolution documented in the literature. IB have made substantial contributions to the conception and design of the work; and the acquisition and interpretation of data; AND to have approved the submitted version (and any substantially modified version that involves the author's contribution to the study); AND to have agreed both to be personally accountable for the author's own contributions and to ensure that questions related to the accuracy or integrity of any part of the work, even ones in which the author was not personally involved, are appropriately investigated, resolved, and the resolution documented in the literature. GD have made substantial contributions to the conception and design of the work; and interpretation of data; and have drafted the work or substantively revised it; AND to have approved the submitted version (and any substantially modified version that involves the author's contribution to the study); AND to have agreed both to be personally accountable for the author's own contributions and to ensure that questions related to the accuracy or integrity of any part of the work, even ones in which the author was not personally involved, are appropriately investigated, resolved, and the resolution documented in the literature. MZ have made substantial contributions to the conception and design of the work; and the acquisition, analysis, and interpretation of data; and have drafted the work or substantively revised it; AND to have approved the submitted version (and any substantially modified version that involves the author's contribution to the study); AND to have agreed both to be personally accountable for the author's own contributions and to ensure that questions related to the accuracy or integrity of any part of the work, even ones in which the author was not personally involved, are appropriately investigated, resolved, and the resolution documented in the literature. All authors read and approved the final manuscript.

\section{Funding}

All sponsors to this manuscript had no role in study design, data analysis or interpretation, writing or decision to submit the report.

\section{Availability of data and materials}

The scan datasets generated during the current study are not publicly available due to the fact that the patients who authorized the use of the data for this study were not questioned about the possibility of making these same data public.

\section{Declarations}

Ethics approval and consent to participate

The study includes dataset has received approval from the IRB of the "GERM Groupe d'éthique recherche médicale" under number 00012157.

\section{Consent for publication}

Not applicable.

\section{Competing interests}

R.A, A.DR.,C.R and G.D'A. are employed by Incepto Medical. Other authors have nothing to declare.

\section{Author details}

${ }^{1}$ Department of Medical Imaging, Saint Joseph Hospital, 185 rue Raymond Losserand, 75014 Paris, France. ${ }^{2}$ Sorbonne Université, 75013 Paris, France. ${ }^{3}$ Incepto-Medical, 128 rue La Boétie, Paris, France.

Received: 26 September 2021 Accepted: 23 December 2021

Published online: 24 January 2022 


\section{References}

1. Gale SC, Shafi S, Dombrovskiy VY, Arumugam D, Crystal JS (2014) The public health burden of emergency general surgery in the United States: a 10-year analysis of the Nationwide Inpatient Sample-2001 to 2010. J Trauma Acute Care Surg 77:202-208. https://doi.org/10.1097/TA.00000 00000000362

2. Miller G, Boman J, Shrier I, Gordon PH (2000) Etiology of small bowel obstruction. Am J Surg 180:33-36. https://doi.org/10.1016/S00029610(00)00407-4

3. ten Broek RPG, Krielen P, Di Saverio S et al (2018) Bologna guidelines for diagnosis and management of adhesive small bowel obstruction (ASBO): 2017 update of the evidence-based guidelines from the world society of emergency surgery ASBO working group. World J Emerg Surg 13:24. https://doi.org/10.1186/s13017-018-0185-2

4. Expert Panel on Gastrointestinal Imaging, Chang KJ, Marin D et al (2020) ACR Appropriateness Criteria ${ }^{\circledR}$ suspected small-bowel obstruction. J Am Coll Radiol 17:S305-S314. https://doi.org/10.1016/j.jacr.2020.01.025

5. Zins M, Millet I, Taourel P (2020) Adhesive small bowel obstruction: predictive radiology to improve patient management. Radiology 296:480492. https://doi.org/10.1148/radiol.2020192234

6. Millet I, Boutot D, Faget C et al (2017) Assessment of strangulation in adhesive small bowel obstruction on the basis of combined CT findings: implications for clinical care. Radiology 285:798-808. https://doi.org/10. 1148/radiol.2017162352

7. Makar RA, Bashir MR, Haystead CM et al (2016) Diagnostic performance of MDCT in identifying closed loop small bowel obstruction. Abdom Radiol (NY) 41:1253-1260. https://doi.org/10.1007/s00261-016-0656-4

8. Montagnon E, Cerny M, Cadrin-Chênevert A et al (2020) Deep learning workflow in radiology: a primer. Insights Imaging 11:22. https://doi.org/ 10.1186/s13244-019-0832-5

9. Cheng PM, Tejura TK, Tran KN, Whang G (2018) Detection of high-grade small bowel obstruction on conventional radiography with convolutional neural networks. Abdom Radiol (NY) 43:1120-1127. https://doi.org/10. 1007/s00261-017-1294-1

10. Cheng PM, Tran KN, Whang G, Tejura TK (2019) Refining convolutional neural network detection of small-bowel obstruction in conventional radiography. AJR Am J Roentgenol 212:342-350. https://doi.org/10.2214/ AJR.18.20362

11. Kim DH, Wit H, Thurston M et al (2021) An artificial intelligence deep learning model for identification of small bowel obstruction on plain abdominal radiographs. Br J Radiol 94:20201407. https://doi.org/10.1259/ bjr.20201407

12. Hodel J, Zins M, Desmottes L et al (2009) Location of the transition zone in CT of small-bowel obstruction: added value of multiplanar reformations. Abdom Imaging 34:35-41. https://doi.org/10.1007/ s00261-007-9348-4

13. Youden WJ (1950) Index for rating diagnostic tests. Cancer 3:32-35. https://doi.org/10.1002/1097-0142(1950)3:1\%3c32::aid-cncr282003 0106\%3e3.0.co:2-3

14. Hou L, Samaras D, Kurc TM, Gao Y, Davis JE, Saltz JH (2016) Patch-based convolutional neural network for whole slide tissue image classification. In: 2016 IEEE conference on computer vision and pattern recognition (CVPR). IEEE, Las Vegas, NV, USA, pp 2424-2433

15. Li Z, Zhang L, Liu X, Yuan F, Song B (2019) Diagnostic utility of CT for small bowel obstruction: systematic review and meta-analysis. PLoS One 14:e0226740. https://doi.org/10.1371/journal.pone.0226740

16. Wang DC, Parry CR, Feldman M, Tomlinson G, Sarrazin J, Glanc P (2015) Acute abdomen in the emergency department: is CT a time-limiting factor? AJR Am J Roentgenol 205:1222-1229. https://doi.org/10.2214/AJR. 14.14057

17. Perotte R, Lewin GO, Tambe $U$ et al (2018) Improving emergency department flow: reducing turnaround time for emergent CT scans. AMIA Ann Symp Proc 2018:897

18. Ferris M, Quan S, Kaplan BS et al (2017) The global incidence of appendicitis: a systematic review of population-based studies. Ann Surg 266:237-241. https://doi.org/10.1097/SLA.0000000000002188

19. Sandhu PS, Joe BN, Coakley FV, Qayyum A, Webb EM, Yeh BM (2007) Bowel transition points: multiplicity and posterior location at CT are associated with small-bowel volvulus. Radiology 245:160-167. https:// doi.org/10.1148/radiol.2443061370
20. Redmon J, Farhadi A (2018) YOLOv3: an incremental improvement. arXiv: 180402767 [CS]

21. Rami Reddy SR, Cappell MS (2017) A systematic review of the clinical presentation, diagnosis, and treatment of small bowel obstruction. Curr Gastroenterol Rep 19:28. https://doi.org/10.1007/s11894-017-0566-9

\section{Publisher's Note}

Springer Nature remains neutral with regard to jurisdictional claims in published maps and institutional affiliations.

\section{Submit your manuscript to a SpringerOpen ${ }^{\circ}$ journal and benefit from:}

- Convenient online submission

- Rigorous peer review

- Open access: articles freely available online

- High visibility within the field

- Retaining the copyright to your article

Submit your next manuscript at $\boldsymbol{\nabla}$ springeropen.com 